\title{
Acúmulo de matéria seca e nutrientes em soja, como variável do potencial produtivo ${ }^{1}$
}

\author{
Carlos Hissao Kurihara², Víctor Hugo Alvarez Venegas ${ }^{3}$, Júlio César Lima Neves ${ }^{4}$, Roberto Ferreira de Novais ${ }^{5}$
}

\section{RESUMO}

O critério atualmente adotado para a indicação de adubação da soja, embasado no estabelecimento de níveis críticos, tem permitido a obtenção de produtividades médias em torno de $3.600 \mathrm{~kg} \mathrm{ha}^{-1}$. Contudo, para a obtenção de produtividades mais elevadas e econômicas, sem prejuízos ao equilíbrio ambiental, deve-se visar à definição de modelos quantitativos que permitam estimar a demanda por nutrientes, em função do potencial de produção almejado. Modelos desta natureza vêm sendo estabelecidos no Departamento de Solos da Universidade Federal de Viçosa, para o desenvolvimento de sistemas de recomendação de corretivos e fertilizantes, denominados genericamente de FERTCALC ${ }^{\circledR}$ e NUTRICALC ${ }^{\circledR}$. A partir de um banco de dados, formado pelo monitoramento nutricional de lavouras comerciais em Mato Grosso do Sul, foram estabelecidos modelos matemáticos para a estimativa da demanda nutricional, em função da produtividade e do acúmulo de nutrientes no terceiro trifólio com pecíolo, ou, então, do coeficiente de utilização biológica (CUB). Demonstrou-se que, para uma dada produtividade, o produto da matéria seca da folha índice com os teores de nutrientes, considerados como suficientes para a cultura, resulta no conteúdo nutricional desta, que, por sua vez, apresenta relação com o conteúdo nutricional no caule, folhas, vagens e nos grãos. A quantidade de nutrientes imobilizada na planta de soja também pode ser calculada a partir do quociente entre a produção de grãos e de matéria seca de parte aérea e os valores de CUB estabelecidos. Os métodos propostos permitem estimativa de valores próximos da demanda nutricional pela soja.

Palavras-chave: demanda nutricional, folha índice, coeficiente de utilização biológica.

\section{ABSTRACT \\ Accumulation of dry matter and nutrients in soybean, as a variable of the productive potential}

The criteria currently adopted for soybean fertilizer recommendation, based on the established of critical levels, has allowed obtaining productivity average around $3600 \mathrm{~kg} \mathrm{ha}^{-1}$. However, in order to obtain greater and more profitable yields without harming the environment, it is necessary to establish new quantitative models to estimate correctly the demand for nutrients, depending on the desired production potential. This kind of models has been established in the Department of Soil Science at the Universidade Federal de Viçosa, for the development of recommendation systems of fertilizers generically called FERTCALC ${ }^{\circledR}$ and NUTRICALC ${ }^{\circledR}$. Mathematical models were established from a database formed by nutritional monitoring of commercial crops in Mato Grosso do Sul State, Brazil, in order to estimate the

Recebido para publicação em 30/01/2013 e aprovado em 25/06/2013.

'Este trabalho é parte da Tese de Doutorado do primeiro autor, apresentada ao Departamento de Solos da Universidade Federal de Viçosa. Trabalho financiado pela Embrapa/ CNPq/Fundect.

${ }^{2}$ Engenheiro-Agrônomo, Doutor. Embrapa Agropecuária Oeste, Rodovia BR 163, Km 253,6, Caixa Postal 661, 79804-970, Dourados, Mato Grosso do Sul, Brasil. carlos.kurihara@embrapa.br (autor para correspondência).

${ }^{3}$ Engenheiro-Agrônomo, Doutor. Departamento de Solos, Universidade Federal de Viçosa, Campus Viçosa, Avenida Peter Henry Rolfs, s/n, 36570-000, Viçosa, Minas Gerais, Brasil.vhav@ufv.br

${ }^{4}$ Engenheiro-Agrônomo, Doutor. Departamento de Solos, Universidade Federal de Viçosa, Campus Viçosa, Avenida Peter Henry Rolfs, s/n, 36570-000, Viçosa, Minas Gerais, Brasil. julio_n2003@yahoo.com.br

${ }^{5}$ Engenheiro-Agrônomo, Doutor. Departamento de Solos, Universidade Federal de Viçosa, Campus Viçosa, Avenida Peter Henry Rolfs, s/n, 36570-000, Viçosa, Minas Gerais, Brasil.rfnovais@ufv.br

Rev. Ceres, Viçosa, v. 60, n.5, p. 690-698, set/out, 2013 
nutrient demand. In these mathematical models, the nutrient demand was a function of potential of production and of nutrient accumulation in the third leaflet with petiole or as a function of the coefficient of biological use (CUB). It was found that, given a certain grain yield, the product between the dry matter of the index leaf and the nutrient levels considered as sufficient or great for the soybean crop results in its nutritional status; this last one is related to the nutritional status of stems, petioles, leaflet, pods and grains. The amount of nutrients in soybean plants can also be calculated by the ratio between grain yield and dry matter of shoot and established values of CUB. The proposed methods allow the estimation of values close to the nutritional demand for soybeans.

Key words: nutritional demand, index leaf, coefficient of biological use.

\section{INTRODUÇÃO}

A recomendação de adubação para uma determinada espécie vegetal, geralmente, é realizada com base na diagnose da fertilidade do solo, associada, eventualmente, à diagnose do estado nutricional da cultura, ou do cultivo antecessor, por meio da interpretação dos resultados da análise química de amostras de solo e de tecido vegetal, coletadas na lavoura, respectivamente. Contudo, considerando-se mesmo extrator químico, mesma marcha analítica e condições semelhantes de solo e de cultivo, têm-se constatado diferenças nos teores de nutrientes estabelecidos para as faixas de disponibilidade no solo, em diferentes regiões do País. Para Novais \& Smyth (1999), os teores de nutrientes definidos nas classes de fertilidade do solo ainda se mostram limitantes para a avaliação da sua disponibilidade, pelo fato de não se considerarem diferenças existentes em função da demanda nutricional da espécie vegetal, do ciclo da cultura, ou, mesmo, do potencial produtivo da lavoura. Além disto, no caso específico de $\mathrm{P}$, estes autores salientam ainda que, em geral, os teores são definidos somente em função do teor de argila, desconsiderando-se que o fator capacidade tampão de fosfato também depende da constituição mineralógica. Por esta razão, Alvarez V. et al. (1999) propõem que, no caso do uso do método Mehlich-1 para a extração de fósforo, a interpretação da disponibilidade de $\mathrm{P}$ seja associada ao valor de fósforo remanescente, definido como a concentração de $\mathrm{P}$ da solução de equilíbrio, após agitar durante uma hora a amostra de terra fina seca ao ar com solução de $\mathrm{CaCl}_{2} 10 \mathrm{mmol} \mathrm{L}^{-1}$, contendo $60 \mathrm{mg}$ $\mathrm{L}^{-1}$ de $\mathrm{P}$, na relação 1:10.

Neste contexto, no Departamento de Solos da Universidade Federal de Viçosa, estão sendo desenvolvidos sistemas de recomendação de corretivos e fertilizantes, baseados no balanço entre a quantidade de nutrientes demandada pelas plantas, para um dado potencial produtivo, e a quantidade que deverá ser suprida pelo solo e pelos resíduos orgânicos da cultura antecessora, conforme Oliveira et al. (2005), Santos et al. (2008) e Silva et al. (2009).
Estes sistemas, denominados genericamente de FERTCALC $^{\circledR}$ e NUTRICALC ${ }^{\circledR}$, implicam o desenvolvimento de modelos que permitam, dentre outros fatores, as estimativas da produção de matéria seca e acúmulo de nutrientes nos diferentes órgãos da planta, no intuito de se predizer as quantidades de nutrientes necessárias à obtenção do potencial produtivo estabelecido.

Partindo-se do pressuposto de que todas as premissas inclusas na formulação do sistema estejam corretas e que as variáveis inseridas sejam adequadas, é lícito pensar que a dose de fertilizante recomendada a partir desse sistema, propicie a otimização de seu uso, pela minimização de perdas de nutrientes por volatilização e, ou, lixiviação, e do seu acúmulo excessivo nos tecidos vegetais. Fica evidente, contudo, que este raciocínio é válido para as condições em que os nutrientes são aplicados adequadamente, em relação à fonte, época e forma e o potencial produtivo almejado esteja coerente com as limitações impostas pelos fatores não nutricionais (clima, nível tecnológico e cultural do agricultor, sistema de manejo do solo e adoção de tratos fitossanitários e culturais, dentre outros) característicos para a região ou, para a propriedade agrícola. Dentro destas limitações, estes fatores devem favorecer o desenvolvimento da cultura durante todo o seu ciclo.

Diferentes modelos foram estabelecidos, para as culturas da bananeira (Oliveira et al., 2005), soja (Santos et al., 2008) e abacaxi (Silva et al., 2009), dentre outras, a partir de levantamentos bibliográficos, resultando em um sistema com um embasamento teórico que permite maior confiabilidade das recomendações técnicas para a fertilização da lavoura. No entanto, as predições tornam-se mais eficientes à medida que os valores considerados aceitáveis são substituídos por outros, originados a partir de avaliações a campo, em condições específicas de lavouras comerciais.

Este trabalho foi conduzido com o objetivo de que se estabeleçam modelos matemáticos para a estimativa da demanda nutricional por plantas de soja, a partir da pro- 
dução de matéria seca associada a um dado potencial produtivo almejado, e dos teores de nutrientes nos diversos órgãos, ou, então, do coeficiente de utilização biológica (CUB) estabelecido.

\section{MATERIAL E MÉTODOS}

Foi efetuada a amostragem de plantas de dez cultivares de soja (BR 16, BRS 133, BRS 181, BRS 182, BRSMS Apaiari, CD 201, Embrapa 48, M-Soy 7501, M-Soy 7508 e M-Soy 5942), no ano agrícola 2001/2002, em 28 lavouras comerciais, cultivadas no sistema plantio direto, em três municípios da região sul do Mato Grosso do Sul: Ponta Porã (Latossolo Vermelho-Amarelo distrófico típico e Latossolo Vermelho distrófico típico), Dourados (Latossolo Vermelho eutroférrico típico e Latossolo Vermelho distroférrico) e Maracaju (Latossolo Vermelho distroférrico). Em cada talhão, procedeu-se à demarcação de uma área representativa com cerca de $2500 \mathrm{~m}^{2}$ (50 x 50 $\mathrm{m})$, onde foram amostradas 30 plantas nos estádios de desenvolvimento R2, R4, R6 e R8 (Fehr \& Caviness, 1977), correspondentes às fases de pleno florescimento, vagem formada, semente cheia e maturidade completa, respectivamente. No estádio R8, o rendimento de grãos, corrigido para umidade de $13 \%$, foi determinado a partir da amostragem de três linhas de cultivo, com $2 \mathrm{~m}$ de comprimento cada, em quatro locais determinados aleatoriamente.

Em laboratório, cada amostra de planta foi separada em caule, pecíolo, trifólio, terceiro pecíolo e terceiro trifólio (nos estádios R2 e R4), vagem (R4, R6 e R8) e grãos (R6 e R8). O material vegetal foi submetido à lavagem sequencial com água, solução ácida $\left(\mathrm{HCl} 0,1 \mathrm{~mol} \mathrm{~L}^{-1}\right)$ e água destilada, conforme Martinez et al. (1999). Após a secagem, em estufa de circulação forçada de ar, a $65^{\circ} \mathrm{C}$, por $72 \mathrm{~h}$, procedeu-se à pesagem e moagem em moinho tipo Wiley, passando-se a amostra em peneira com malha de $0,85 \mathrm{~mm}$ (20 mesh).

Procedeu-se à caracterização química de teores de macro e micronutrientes nas amostras de tecido vegetal, sendo a determinação de $\mathrm{N}$ efetuada pelo método semimicro-Kjeldahl. Os demais nutrientes foram extraídos das amostras por meio de digestão nítrico-perclórica e determinados por espectrometria de emissão ótica, em plasma induzido (ICP-OES), regulado para potência de $1.300 \mathrm{~W}$ e para as seguintes taxas de fluxo: $15 \mathrm{~L} \mathrm{~min}^{-1}$ de argônio, 0,5 $\mathrm{L} \mathrm{min}^{-1}$ de $\mathrm{N}, 0,6 \mathrm{~L} \mathrm{~min}^{-1}$ de ar comprimido e 2,5 $\mathrm{L} \mathrm{min}^{-1}$ de solução de leitura, conforme Kurihara et al. (2003).

Os teores de macro e micronutrientes foram expressos em $\mathrm{g} \mathrm{kg}^{-1}$ e mg kg${ }^{-1}$, respectivamente. $\mathrm{O}$ conteúdo de macro $\left(\mathrm{kg} \mathrm{ha}^{-1}\right)$ e micronutrientes $\left(\mathrm{g} \mathrm{ha}^{-1}\right)$, nos diferentes órgãos da planta, foi estimado pelo produto do teor com o rendimento de matéria seca (em kg ha ${ }^{-1}$ ) e um fator de ajuste de 0,001 .

A partir do quociente entre a quantidade de matéria seca, produzida pela parte aérea (caule, pecíolos, folhas e vagens) e grãos de soja e a quantidade acumulada de nutriente, obtiveram-se os valores médios de coeficiente de utilização biológica (CUB), expressos em kg kg-1 (Oliveira et al., 2005, Santos et al., 2008 e Silva et al. 2009), conforme exemplo a seguir, considerando-se produção de matéria seca de $3.000 \mathrm{~kg} \mathrm{ha}^{-1}$ de grãos de soja e acúmulo de $180 \mathrm{~kg} \mathrm{ha}^{-1}$ do nutriente:

CUB $=\frac{3.000 \mathrm{~kg} / \text { ha matéria seca produzida }}{180 \mathrm{~kg} / \text { ha nutriente acumulado }}=16,7 \mathrm{~kg}$
grãos $\mathrm{kg}^{-1}$ de nutriente acumulado

Desta forma, o CUB pode ser definido como a quantidade de matéria seca produzida por unidade de nutriente acumulado, e representa uma medida da eficiência da planta em converter nutriente absorvido em matéria seca (Barros et al., 1995).

A partir dos resultados obtidos, efetuou-se o ajuste de modelos de regressão, usando-se o software SAEG v. 9.1 - 2007, para produção de matéria seca de diferentes órgãos da planta de soja, em quatro estádios de desenvolvimento, em função do rendimento de grãos, e para conteúdo de nutriente nos diferentes órgãos, nos estádios R4 (caule, pecíolo e trifólios), R6 (caule, pecíolo, trifólios e vagens) e R8 (grãos), em função do conteúdo deste no terceiro trifólio com pecíolo, no estádio R2. Dentre os modelos de regressão testados (linear, quadrático, cúbico, raiz quadrada, potencial, exponencial e hiperbólico), definiu-se, para cada conjunto de variáveis, aquele com maior coeficiente de determinação, cujos estimadores de parâmetros da equação eram significativos a, no mínimo, $5 \%$ de probabilidade. Salienta-se que, os modelos de regressão estabelecidos são válidos para uma faixa de produtividade de grãos entre 2.813 e $4.810 \mathrm{~kg} \mathrm{ha}^{-1}$, obtida nos talhões avaliados.

\section{RESULTADOS E DISCUSSÃO}

Na Tabela 1, são apresentados os modelos ajustados para a estimativa de produção de matéria seca dos diferentes órgãos da planta (caule, pecíolos, trifólios e vagens), e da parte aérea como um todo, nos quatro estádios de desenvolvimento avaliados, em função do rendimento de grãos. A produção de matéria seca de vagens, no estádio R4, não apresentou ajuste adequado com o rendimento de grãos, dado o intenso acúmulo de matéria seca neste órgão durante este período (Bianco et al., 2007). Este acúmulo é devido, principalmente, ao desenvolvimento das vagens em tamanho e ocorre até o início do estádio R6, a partir do qual permanece praticamente inalterado (Lazarini et al., 2000). Salienta-se que, as coletas de amostras não ocorreram no mesmo número de dias após a emergência das plantas, em virtude de diferenças nas datas de semeadura e nos ciclos de desenvolvimento 
vegetativo dos cultivares. De acordo com Cruz et al. (2010), a massa de matéria seca total da soja é influenciada, de forma mais pronunciada, pela época de semeadura, do que pelo ciclo de maturação do cultivar. Portanto, pequenas diferenças temporais na amostragem podem ter originado grande variabilidade na produção de matéria seca. Da mesma forma, na fase de maturação completa dos grãos (R8), as plantas encontravam-se praticamente senescentes, e, os poucos trifólios, que ainda não haviam caído, apresentaram grande variabilidade na produção de matéria seca.

A partir destes modelos de regressão, uma vez definido o potencial produtivo almejado para determinada lavoura, pode-se estimar a produção de matéria seca da parte aérea de plantas de soja, ou de seus órgãos, separadamente (Tabela 1), em distintos estádios de desenvolvimento. Os dados de matéria seca produzida nos órgãos das plantas de soja, por sua vez, podem ser utilizados para se estimar a quantidade de cada nutriente requerida nos estádios reprodutivos da cultura. Esta inferência, no entanto, é válida somente dentro dos limites da faixa de abrangência das produtividades, entre 2.813 e $4.810 \mathrm{~kg}$ $\mathrm{ha}^{-1}$ obtidas nos talhões amostrados neste trabalho. Destaca-se que todos os talhões avaliados apresentavam histórico de boa fertilidade do solo e de manejo adequado de pragas, doenças e infestação de plantas daninhas.

Uma forma de se calcular a exigência nutricional da soja consiste na estimativa da massa de matéria seca de folha índice (terceiro trifólio com pecíolo), em função do potencial produtivo almejado (Tabela 1 ). O produto da massa de matéria seca de folha índice, com os teores médios de nutrientes considerados como suficientes para a cultura (entre os teores críticos e ótimos), estimados por um método de diagnose do estado nutricional (Reis Jr \& Monnerat, 2003; Kurihara, 2004; Urano et al., 2007; Serra et al., 2010), resulta no conteúdo de nutrientes da folha índice. A partir disto, pode-se proceder à estimativa do conteúdo nutricional no caule, nos pecíolos, nos trifólios, nas vagens e nos grãos, nos estádios de desenvolvimento de máximo acúmulo na parte aérea (Tabelas 2 a 5), que, de acordo com Kurihara (2004), ocorre entre os estádios R4 (para N, K, B e Fe) e R6 (para P, Ca, Mg, S, Cu, Mn e $\mathrm{Zn}$ ). Por esta razão, optou-se por ajustar modelos para ambos, no intuito de se permitir o cálculo da demanda nutricional pela soja, associado ao potencial produtivo estabelecido. Nas Tabelas 2 a 5, constata-se que, em geral, o modelo com melhor ajuste entre as duas variáveis independentes avaliadas foi o potencial, sendo que, em alguns casos também se definiu ajuste de equação linear, quadrática, exponencial ou hiperbólica. Ressalta-se que foram estabelecidos os modelos de regressão que apresentaram os maiores coeficientes de determinação, com estimadores de parâmetros da equação significativos a 1 ou $5 \%$ de probabilidade. Em muitos casos, a dispersão dos dados existente nas duas variáveis independentes, utilizadas na análise de regressão, resultou em modelos com baixo coeficiente de determinação, principalmente para micronutrientes. Esta dispersão provavelmente é decorrente da forma de amostragem adotada, efetuada em 28 lavouras comerciais, cultivadas no sistema plantio direto, em três municípios da região sul do Mato Grosso do Sul, incluindo-se talhões distintos, quanto ao ciclo de desenvolvimento dos cultivares e, à época de semeadura.

Tabela 1. Equações de regressão para produção de matéria seca $(\mathrm{t} / \mathrm{ha})$ de caule $(\mathrm{c})$, pecíolos $(\mathrm{p})$, trifólios $(\mathrm{t})$, terceiro trifólio (tt), terceiro trifólio com pecíolo (ttp), vagens (v) e parte aérea ${ }^{1}$ (pa), em diferentes estádios de desenvolvimento (R2, R4, R6 e R8), em função de rendimento de grãos $\left(\mathrm{RG}, \mathrm{t} \mathrm{ha}^{-1}\right)$ de soja

\begin{tabular}{|c|c|c|c|}
\hline Equação ${ }^{2}$ & $\mathrm{R}^{2}$ & Equação ${ }^{2}$ & $\mathrm{R}^{2}$ \\
\hline - - & - - - & $-\ldots-\ldots$ R4 & -- \\
\hline$\hat{c}_{2}=0,110 \mathrm{e}^{0,610^{* * *} \mathrm{rg}}$ & 0,715 & $\hat{\mathrm{c}}_{4}=0,217 \mathrm{e}^{0,611 * * \mathrm{rg}}$ & 0,656 \\
\hline$\hat{\mathrm{p}}_{2}=0,050 \mathrm{e}^{0,617 * *_{\mathrm{rg}}}$ & 0,716 & $\hat{\mathrm{p}}_{4}=0,062 \mathrm{e}^{0,710^{* *} \mathrm{rg}}$ & 0,769 \\
\hline$\hat{\mathrm{t}}_{2}=0,137 \mathrm{e}^{0,576^{* *} \mathrm{rg}}$ & 0,679 & $\hat{\mathrm{t}}_{4}=0,185 \mathrm{e}^{0,597 * * \mathrm{rg}}$ & 0,625 \\
\hline$\hat{\mathrm{tt}}_{2}=0,027 \mathrm{e}^{0,542 * \mathrm{rg}_{\mathrm{rg}}}$ & 0,679 & $\hat{\mathrm{tt}}_{4}=0,044 \mathrm{e}^{0,443^{* *} \mathrm{rg}}$ & 0,532 \\
\hline$\hat{\mathrm{ttp}}_{2}=0,044 \mathrm{e}^{0,524 * * \mathrm{rg}}$ & 0,650 & $\hat{\mathrm{ttp}}_{4}=0,071 \mathrm{e}^{0,443 * * \mathrm{rg}}$ & 0,597 \\
\hline \multirow[t]{2}{*}{$\hat{\mathrm{p}} \mathrm{a}_{2}=0,333 \mathrm{e}^{0,588 * * \mathrm{rg}}$} & 0,727 & $\hat{\mathrm{v}}_{4}=0,008 \mathrm{e}^{2,374 * \mathrm{rg}_{\mathrm{rg}}}$ & 0,392 \\
\hline & & $\hat{\mathrm{p}} \mathrm{a}_{4}=0,506 \mathrm{e}^{0,615^{* *} \mathrm{rg}}$ & 0,704 \\
\hline - . L R6 - & --- & $\ldots \ldots$ &.- \\
\hline$\hat{\mathrm{c}}_{6}=0,338 \mathrm{e}^{0,568 * * \mathrm{rg}}$ & 0,705 & $\hat{\mathrm{c}}_{8}=0,255 \mathrm{e}^{0,570^{* *} \mathrm{rg}}$ & 0,642 \\
\hline$\hat{\mathrm{p}}_{6}=0,144 \mathrm{e}^{0,548^{* * \mathrm{r}_{\mathrm{rg}}}}$ & 0,809 & $\hat{\mathrm{p}}_{8}=0,008 \mathrm{e}^{0,919 * *_{\mathrm{rg}}}$ & 0,555 \\
\hline$\hat{\mathrm{t}}_{6}=0,175 \mathrm{e}^{0,601 * * \mathrm{rg}}$ & 0,738 & $\hat{\mathrm{t}}_{8}=1 /(21,5-3,72 * \mathrm{rg})$ & 0,210 \\
\hline$\hat{\mathrm{v}}_{6}=0,074 \mathrm{e}^{0,742 * \mathrm{rg}_{\mathrm{rg}}}$ & 0,578 & $\hat{\mathrm{v}}_{8}=0,089 \mathrm{e}^{0,701 * * \mathrm{rg}}$ & 0,702 \\
\hline$\hat{\mathrm{pa}} \mathrm{a}_{6}=0,717 \mathrm{e}^{0,611^{* *} \mathrm{rg}}$ & 0,751 & $\hat{\mathrm{p}} \mathrm{a}_{8}=0,360 \mathrm{e}^{0,635 * *_{\mathrm{rg}}}$ & 0,663 \\
\hline
\end{tabular}

${ }^{1}$ A matéria seca de parte aérea refere-se ao somatório da matéria seca de caule, pecíolos, trifólios e vagens (nos estádios R4, R6 e R8).

${ }^{2}$ Os modelos ajustados são válidos para uma faixa de rendimento de grãos entre 2,813 e 4,810 t ha-1. 
O procedimento foi adotado pelo fato de ter-se como objetivo o ajuste de modelos de regressão válidos para condições gerais de cultivo de soja, conforme apresentado nas Tabelas 2 a 5. Acredita-se que o ajuste de modelos específicos para um cultivar e uma época de semeadura possa resultar em equações de regressão com coeficientes de determinação mais elevados; no entanto, perderse-ia o foco do desenvolvimento de um sistema preditivo das quantidades de nutrientes necessárias à obtenção do potencial produtivo estabelecido.

Na Tabela 6, são apresentados valores médios de coeficientes de utilização biológica (CUB) de macro e micronutrientes para a parte aérea (estádios de desenvolvimento R4 e R6) e para os grãos (R8). A partir do quociente entre a produção de grãos ou de matéria seca de parte aérea, estimada em função de uma definida produtividade de grãos (Tabela 1), e os valores de coeficiente de utilização biológica (CUB) estabelecidos (Tabela 6), tem- se uma alternativa para o cálculo da quantidade de nutrientes imobilizada na planta de soja. Observa-se que, os valores de eficiência de aproveitamento apresentaram variação entre os estádios R4 e R6, principalmente, para N, K, B, Fe e Zn. Por esta razão, sugere-se a adoção de valores referentes ao estádio de desenvolvimento em que cada nutriente apresenta o acúmulo máximo, ou seja, R4 para N, K, B e Fe, e R6 para P, Ca, Mg, S, Cu, Mn e Zn (Kurihara, 2004).

Para uma produtividade esperada de 4,0 t ha ${ }^{-1}$ de grãos de soja, os modelos de regressão apresentados na Tabela 1 permitem estimar uma produção de 3,278; 1,289; 1 ,937 e 1,440 t ha $^{-1}$ de caule, pecíolos, trifólios e vagens, respectivamente, no estádio de desenvolvimento R6. O somatório da massa de matéria seca, calculado para os diferentes órgãos da planta, de 7,944 t ha-1 , mostra-se bastante próximo do valor estimado para toda a parte aérea $\left(8,259 \mathrm{t} \mathrm{ha}^{-1}\right)$, pela equação $\hat{\text { pa }}{ }_{6}=0,717 \mathrm{e}^{0,611^{* *} \mathrm{rg}}$, apresentada na Tabela 1 .

Tabela 2. Equações de regressão para o conteúdo de macro $\left(\mathrm{kg} \mathrm{ha}^{-1}\right)$ e micronutriente $\left(\mathrm{g} \mathrm{ha}^{-1}\right)$ no caule $(\mathrm{c})$, nos estádios de desenvolvimento R4 (4) e R6 (6), em função do conteúdo deste no terceiro trifólio com pecíolo (ttp), no estádio R2, na cultura da soja

\begin{tabular}{|c|c|c|}
\hline & & $\mathrm{R}^{2}$ \\
\hline$\hat{\mathrm{n}}_{\mathrm{c} 4}=3,23 * * \mathrm{n}_{\mathrm{ttp}} 0,953$ & $\forall 4,3 \leq \mathrm{n}_{\mathrm{ttp}} \leq 20 \mathrm{~kg} \mathrm{ha}^{-1}$ & 0,802 \\
\hline$\hat{\mathrm{p}}_{\mathrm{c} 6}=5,50 * * \mathrm{p}_{\mathrm{ttp}} 1,27$ & $\forall 0,23 \leq \mathrm{p}_{\text {ttp }} \leq 1,5 \mathrm{~kg} \mathrm{ha}^{-1}$ & 0,857 \\
\hline$\hat{\mathrm{k}}_{\mathrm{c} 4}=5,29 * * \mathrm{k}_{\mathrm{ttp}}^{0,973}$ & $\forall 3,1 \leq \mathrm{k}_{\mathrm{ttp}} \leq 14 \mathrm{~kg} \mathrm{ha}^{-1}$ & 0,674 \\
\hline$\hat{\mathrm{ca}} \mathrm{c}_{\mathrm{c} 6}=3,46^{* *} \mathrm{ca}_{\mathrm{ttp}} 0,995$ & $\forall 0,99 \leq \mathrm{ca}_{\text {ttp }} \leq 6,2 \mathrm{~kg} \mathrm{ha}^{-1}$ & 0,818 \\
\hline$\hat{\mathrm{m}} \mathrm{c}_{\mathrm{c} 6}=2,31 \cdot \mathrm{e}^{1,02 * * \mathrm{mg}_{\mathrm{ttp}}}$ & $\forall 0,49 \leq \mathrm{mg}_{\text {ttp }} \leq 2,1 \mathrm{~kg} \mathrm{ha}^{-1}$ & 0,787 \\
\hline$\hat{\mathrm{s}}_{\mathrm{c} 6}=3,55^{* *} \mathrm{~s}_{\mathrm{ttp}} 0,813$ & $\forall 0,21 \leq \mathrm{s}_{\text {ttp }} \leq 1,7 \mathrm{~kg} / \mathrm{ha}$ & 0,603 \\
\hline$\hat{\mathrm{b}}_{\mathrm{c} 4}=13,5+3,16^{* *} \mathrm{~b}_{\mathrm{ttp}}$ & $\forall 5,2 \leq \mathrm{b}_{\mathrm{ttp}} \leq 19 \mathrm{~g} \mathrm{ha}^{-1}$ & 0,560 \\
\hline$\hat{\mathrm{cu}} \mathrm{c}_{\mathrm{c} 6}=25,8 * * \mathrm{cu}_{\mathrm{tp}}, 0,438$ & $\forall 1,1 \leq \mathrm{cu}_{\mathrm{tpp}} \leq 11 \mathrm{~g} \mathrm{ha}^{-1}$ & 0,402 \\
\hline$\hat{\mathrm{fe}}_{\mathrm{c} 4}=1,59^{* *} \mathrm{fe}_{\mathrm{ttp}} 1,33$ & $\forall 13 \leq \mathrm{fe}_{\mathrm{ttp}} \leq 39 \mathrm{~g} \mathrm{ha}^{-1}$ & 0,781 \\
\hline$\hat{\mathrm{z}} \mathrm{n}_{\mathrm{c} 6}=-1,90+2,10^{* *} \mathrm{zn}_{\mathrm{ttp}}$ & $\forall 4,2 \leq \mathrm{zn}_{\text {ttp }} \leq 30 \mathrm{~g} \mathrm{ha}^{-1}$ & 0,655 \\
\hline
\end{tabular}

Tabela 3. Equações de regressão para o conteúdo de macro $\left(\mathrm{kg} \mathrm{ha}^{-1}\right)$ e micronutriente $\left(\mathrm{g} \mathrm{ha}^{-1}\right)$ nos pecíolos $(\mathrm{p})$, nos estádios de desenvolvimento R4 (4) e R6 (6), em função do conteúdo deste no terceiro trifólio com pecíolo (ttp), no estádio R2, na cultura da soja

\begin{tabular}{|c|c|c|}
\hline & & $\mathrm{R}^{2}$ \\
\hline$\hat{\mathrm{n}}_{\mathrm{p} 4}=0,875^{* *} \mathrm{n}_{\mathrm{ttp}} 1,04$ & $\forall 4,3 \leq \mathrm{n}_{\text {ttp }} \leq 20 \mathrm{~kg} \mathrm{ha}^{-1}$ & 0,812 \\
\hline$\hat{\mathrm{p}}_{\mathrm{p} 6}=2,03 * * \mathrm{p}_{\mathrm{ttp}}, 26$ & $\forall 0,23 \leq \mathrm{p}_{\text {ttp }} \leq 1,5 \mathrm{~kg} \mathrm{ha}^{-1}$ & 0,841 \\
\hline$\hat{\mathrm{k}}_{\mathrm{p} 4}=4,52 * * \mathrm{k}_{\mathrm{ttp}} 0,937$ & $\forall 3,1 \leq \mathrm{k}_{\mathrm{ttp}} \leq 14 \mathrm{~kg} \mathrm{ha}^{-1}$ & 0,585 \\
\hline$\hat{\mathrm{ca}} \mathrm{p}_{\mathrm{p} 6}=5,31 * * \mathrm{ca}_{\mathrm{ttp}}{ }^{0,876}$ & $\forall 0,99 \leq \mathrm{ca}_{\mathrm{ttp}} \leq 6,6 \mathrm{~kg} \mathrm{ha}^{-1}$ & 0,722 \\
\hline$\hat{\mathrm{m}} \mathrm{g}_{\mathrm{p} 6}=3,38 * \mathrm{mg}_{\mathrm{ttp}} 1,15$ & $\forall 0,49 \leq \mathrm{mg}_{\text {ttp }} \leq 2,1 \mathrm{~kg} \mathrm{ha}^{-1}$ & 0,694 \\
\hline$\hat{\mathrm{s}}_{\mathrm{p} 6}=0,847 * * \mathrm{~s}_{\mathrm{ttp}} 0,589$ & $\forall 0,21 \leq \mathrm{s}_{\mathrm{ttp}} \leq 1,9 \mathrm{~kg} \mathrm{ha}^{-1}$ & 0,385 \\
\hline$\hat{\mathrm{b}}_{\mathrm{p} 4}=5,15^{* *} \mathrm{~b}_{\mathrm{ttp}}^{0,672}$ & $\forall 5,2 \leq \mathrm{b}_{\mathrm{ttp}} \leq 19 \mathrm{~g} \mathrm{ha}^{-1}$ & 0,376 \\
\hline$\hat{\mathrm{cu}_{\mathrm{n}}^{r}}=1,44+2,06^{*} * \mathrm{cu}_{\mathrm{ttp}}$ & $\forall 1,2 \leq \mathrm{cu}_{\text {ttp }} \leq 7,6 \mathrm{~g} \mathrm{ha}^{-1}$ & 0,442 \\
\hline$\hat{\mathrm{fe}}_{\mathrm{p} 4}=0,617^{* *} \mathrm{fe}_{\mathrm{ttp}}{ }^{1,41}$ & $\forall 18 \leq \mathrm{fe}_{\mathrm{ttp}} \leq 39 \mathrm{~g} \mathrm{ha}^{-1}$ & 0,499 \\
\hline$\hat{\mathrm{z}} \mathrm{n}_{\mathrm{p} 6}=0,751^{* *} \mathrm{zn}_{\mathrm{tp}}, 1,14$ & $\forall 6,9 \leq \mathrm{zn}_{\mathrm{ttp}} \leq 21 \mathrm{~g} \mathrm{ha}^{-1}$ & 0,432 \\
\hline
\end{tabular}

Rev. Ceres, Viçosa, v. 60, n.5, p. 690-698, set/out, 2013 
Esta constatação é confirmada pelo ajuste de um modelo de regressão linear com intercepto tendendo a zero e coeficiente angular tendendo à unidade $(\hat{\mathrm{y}}=-0,0814+$ $1,0499 * * x$, com $\left.\mathrm{R}^{2}=1,00\right)$, para uma série de valores de total de biomassa, calculada para diferentes órgãos em função de massa de matéria seca estimada para toda a parte aérea, considerando-se uma amplitude de potencial produtivo de 2 a $5 \mathrm{t} \mathrm{ha}^{-1}$ de grãos.

A partir da equação $\hat{t t p}_{2}=0,044 \mathrm{e}^{0,524 * \mathrm{rg} g}$, apresentada na Tabela 1, pode-se estimar que, para o potencial produtivo de 4,0 t ha-1 de grãos, há a produção de $0,358 \mathrm{t} \mathrm{ha}^{-1} \mathrm{de}$ matéria seca de terceiro trifólio com pecíolo, no estádio de

Tabela 4. Equações de regressão para o conteúdo de macro $\left(\mathrm{kg} \mathrm{ha}^{-1}\right)$ e micronutriente $\left(\mathrm{g} \mathrm{ha}^{-1}\right)$ nos trifólios (t), nos estádios de desenvolvimento R4 (4) e R6 (6), em função do conteúdo deste no terceiro trifólio com pecíolo (ttp), no estádio R2, na cultura da soja

\begin{tabular}{|c|c|c|}
\hline \multicolumn{2}{|c|}{ Equa๔ão } & \multirow{2}{*}{$\frac{\mathrm{R}^{2}}{0,763}$} \\
\hline$\hat{\mathrm{n}}_{\mathrm{t} 4}=10,4^{*} * \mathrm{n}_{\mathrm{ttp}} 0,829$ & $\forall 4,3 \leq \mathrm{n}_{\mathrm{ttp}} \leq 20 \mathrm{~kg} \mathrm{ha}^{-1}$ & \\
\hline$\hat{\mathrm{p}}_{\mathrm{t} 6}=5,25^{* *} \mathrm{p}_{\mathrm{ttp}} 0,908$ & $\forall 0,23 \leq \mathrm{p}_{\text {ttp }} \leq 1,5 \mathrm{~kg} \mathrm{ha}^{-1}$ & 0,845 \\
\hline$\hat{\mathrm{k}}_{\mathrm{t} 4}=5,25^{* *} \mathrm{k}_{\mathrm{ttp}}{ }^{0,821}$ & $\forall 3,1 \leq \mathrm{k}_{\mathrm{ttp}} \leq 14 \mathrm{~kg} \mathrm{ha}^{-1}$ & 0,780 \\
\hline$\hat{\mathrm{k}}_{\mathrm{t} 6}=3,75^{* *} \mathrm{k}_{\mathrm{ttp}} 0,882$ & $\forall 3,1 \leq \mathrm{k}_{\mathrm{ttp}} \leq 14 \mathrm{~kg} \mathrm{ha}^{-1}$ & 0,871 \\
\hline $\mathrm{ca}_{\mathrm{t} 6}=6,15 * * \mathrm{ca}_{\mathrm{ttp}}{ }^{1,07}$ & $\forall 0,99 \leq \mathrm{ca}_{\mathrm{ttp}} \leq 6,6 \mathrm{~kg} \mathrm{ha}^{-1}$ & 0,817 \\
\hline$\hat{\mathrm{m}} \mathrm{g}_{\mathrm{t} 6}=-1,45+4,92 * * \mathrm{mg}_{\mathrm{ttp}}$ & $\forall 0,49 \leq \mathrm{mg}_{\text {ttp }} \leq 2,1 \mathrm{~kg} \mathrm{ha}^{-1}$ & 0,831 \\
\hline$\hat{\mathrm{s}}_{\mathrm{t} 6}=5,14 * *_{\mathrm{ttp}} 0,394$ & $\forall 0,21 \leq \mathrm{s}_{\text {ttp }} \leq 1,9 \mathrm{~kg} \mathrm{ha}^{-1}$ & 0,534 \\
\hline$\hat{\mathrm{b}}_{\mathrm{t} 4}=4,86^{* *} \mathrm{~b}_{\mathrm{ttp}}^{0,938}$ & $\forall 5,2 \leq \mathrm{b}_{\mathrm{ttp}} \leq 19 \mathrm{~g} \mathrm{ha}^{-1}$ & 0,566 \\
\hline$\hat{\mathrm{c}} \mathrm{u}_{\mathrm{t} 6}=4,85+1,82 * * \mathrm{cu}_{\mathrm{tp}}$ & $\forall 1,1 \leq \mathrm{cu}_{\mathrm{ttp}} \leq 11 \mathrm{~g} \mathrm{ha}^{-1}$ & 0,770 \\
\hline$\hat{\mathrm{fe}}_{\mathrm{t} 4}=20,3^{* *} \mathrm{fe}_{\mathrm{ttp}}{ }^{0,961}$ & $\forall 13 \leq \mathrm{fe}_{\mathrm{ttp}} \leq 56 \mathrm{~g} \mathrm{ha}^{-1}$ & 0,716 \\
\hline$\hat{\mathrm{m}} \mathrm{t}_{\mathrm{t} 6}=258-1.757 * * 1 / \mathrm{mn}_{\mathrm{ttp}}$ & $\forall 8,2 \leq \mathrm{mn}_{\mathrm{ttp}} \leq 56 \mathrm{~g} \mathrm{ha}^{-1}$ & 0,674 \\
\hline$\hat{\mathrm{z}} \mathrm{n}_{\mathrm{t} 6}=9,62 * *_{\mathrm{zn}} \mathrm{n}_{\mathrm{tp}} 0,666$ & $\forall 4,2 \leq \mathrm{zn}_{\text {ttp }} \leq 30 \mathrm{~g} \mathrm{ha}^{-1}$ & 0,550 \\
\hline
\end{tabular}

Tabela 5. Equações de regressão para o conteúdo de macro $\left(\mathrm{kg} \mathrm{ha}^{-1}\right)$ e micronutriente $\left(\mathrm{g} \mathrm{ha}^{-1}\right)$ nas vagens (v) e nos grãos $(\mathrm{g})$, nos estádios de desenvolvimento R6 (6) e R8 (8), respectivamente, em função do conteúdo deste no terceiro trifólio com pecíolo (ttp), no estádio R2, na cultura da soja

\begin{tabular}{|c|c|c|}
\hline Equação $^{1}$ & & $\mathrm{R}^{2}$ \\
\hline$\hat{\mathrm{n}}_{\mathrm{V} 6}=2,70 * * \mathrm{n}_{\mathrm{TTP}} 0,917$ & $\forall 4,3 \leq \mathrm{N}_{\mathrm{TTP}} \leq 20 \mathrm{~kg} \mathrm{ha}^{-1}$ & 0,755 \\
\hline$\hat{\mathrm{n}}_{\mathrm{g} 8}=69,3 * * \mathrm{n}_{\mathrm{TTP}} 0,459$ & $\forall 8,5 \leq \mathrm{N}_{\mathrm{TTP}} \leq 20 \mathrm{~kg} \mathrm{ha}^{-1}$ & 0,525 \\
\hline$\hat{\mathrm{p}}_{\mathrm{v} 6}=4,28 * * \mathrm{p}_{\mathrm{ttp}} 0,936$ & $\forall 0,23 \leq \mathrm{p}_{\mathrm{ttp}} \leq 1,5 \mathrm{~kg} \mathrm{ha}^{-1}$ & 0,776 \\
\hline$\hat{\mathrm{p}}_{\mathrm{g} 8}=23,3 * * \mathrm{P}_{\mathrm{TTP}} 0,324$ & $\forall 0,23 \leq \mathrm{P}_{\mathrm{TTP}} \leq 1,5 \mathrm{~kg} \mathrm{ha}^{-1}$ & 0,594 \\
\hline$\hat{\mathrm{k}}_{\mathrm{v} 6}=0,312+0,441 * * \mathrm{k}_{\mathrm{tp}}$ & $\forall 3,1 \leq \mathrm{k}_{\mathrm{ttp}} \leq 13 \mathrm{~kg} \mathrm{ha}^{-1}$ & 0,735 \\
\hline$\hat{\mathrm{k}}_{\mathrm{g} 8}=23,9 * * \mathrm{k}_{\mathrm{TTP}}, 0,399$ & $\forall 3,1 \leq \mathrm{K}_{\mathrm{TTP}} \leq 14 \mathrm{~kg} \mathrm{ha}^{-1}$ & 0,655 \\
\hline$\hat{\mathrm{ca}} \mathrm{v}_{\mathrm{v} 6}=3,09 * * \mathrm{ca}_{\mathrm{ttp}}{ }^{0,898}$ & $\forall 0,99 \leq \mathrm{ca}_{\mathrm{ttp}} \leq 6,6 \mathrm{~kg} \mathrm{ha}^{-1}$ & 0,869 \\
\hline$\hat{\mathrm{ca}} \mathrm{a}_{\mathrm{g} 8}=6,28 * * \mathrm{ca}_{\mathrm{ttp}} 0,283$ & $\forall 0,99 \leq \mathrm{ca}_{\text {ttp }} \leq 6,6 \mathrm{~kg} \mathrm{ha}^{-1}$ & 0,369 \\
\hline$\hat{\mathrm{m}} \mathrm{v}_{\mathrm{v} 6}=5,22 * * \mathrm{mg}_{\mathrm{ttp}} 1,12$ & $\forall 0,49 \leq \mathrm{mg}_{\text {ttp }} \leq 2,1 \mathrm{~kg} \mathrm{ha}^{-1}$ & 0,861 \\
\hline$\hat{\mathrm{m}} \mathrm{g}_{\mathrm{g} 8}=2,34+11,8^{* *} \mathrm{mg}_{\mathrm{ttp}}-3,73^{* *} \mathrm{mg}_{\mathrm{ttp}}{ }^{2}$ & $\forall 0,49 \leq \mathrm{mg}_{\mathrm{ttp}} \leq 2,0 \mathrm{~kg} \mathrm{ha}^{-1}$ & 0,610 \\
\hline$\hat{\mathrm{s}}_{\mathrm{v} 6}=2,19 * *_{\mathrm{ttp}} 0,479$ & $\forall 0,21 \leq \mathrm{s}_{\text {ttp }} \leq 1,9 \mathrm{~kg} \mathrm{ha}^{-1}$ & 0,432 \\
\hline$\hat{\mathrm{b}}_{\mathrm{v} 6}=6,47+3,50 * * \mathrm{~b}_{\mathrm{ttp}}$ & $\forall 5,2 \leq \mathrm{b}_{\text {ttp }} \leq 19 \mathrm{~g} \mathrm{ha}^{-1}$ & 0,506 \\
\hline$\hat{\mathrm{b}}_{\mathrm{g} 8}=39,8 * * \mathrm{~b}_{\mathrm{ttp}} 0,373$ & $\forall 5,2 \leq \mathrm{b}_{\mathrm{ttp}} \leq 19 \mathrm{~g} \mathrm{ha}^{-1}$ & 0,446 \\
\hline$\hat{\mathrm{cu}} \mathrm{v}_{\mathrm{v} 6}=3,67 * * \mathrm{cu}_{\mathrm{ttp}} 0,706$ & $\forall 1,1 \leq \mathrm{cu}_{\mathrm{ttp}} \leq 8,0 \mathrm{~g} \mathrm{ha}^{-1}$ & 0,619 \\
\hline$\hat{c} u_{\mathrm{g} 8}=-23,3+50,7 * * \mathrm{cu}_{\mathrm{ttp}}$ & $\forall 1,2 \leq \mathrm{cu}_{\mathrm{tp}} \leq 6,1 \mathrm{~g} \mathrm{ha}^{-1}$ & 0,589 \\
\hline$\hat{\mathrm{fe}}_{\mathrm{g} 8}=110+7,02 * * \mathrm{fe}_{\mathrm{ttp}}$ & $\forall 65 \leq \mathrm{fe}_{\mathrm{ttp}} \leq 282 \mathrm{~g} \mathrm{ha}^{-1}$ & 0,622 \\
\hline$\hat{\mathrm{m}}_{\mathrm{v} 6}=8,32 * * \mathrm{mn}_{\mathrm{ttp}} 0,577$ & $\forall 8,2 \leq \mathrm{mn}_{\text {ttp }} \leq 39 \mathrm{~g} \mathrm{ha}^{-1}$ & 0,428 \\
\hline$\hat{\mathrm{m}}_{\mathrm{g} 8}=62,2 * * \mathrm{mn}_{\mathrm{ttp}} 0,250$ & $\forall 8,2 \leq \mathrm{mn}_{\mathrm{ttp}} \leq 39 \mathrm{~g} \mathrm{ha}^{-1}$ & 0,339 \\
\hline $\mathrm{zn}_{\mathrm{v} 6}=8,26+2,10^{* *} \mathrm{zn}_{\mathrm{ttp}}$ & $\forall 4,2 \leq \mathrm{zn}_{\mathrm{ttp}} \leq 21 \mathrm{~g} \mathrm{ha}^{-1}$ & 0,714 \\
\hline$\hat{z} n_{\mathrm{g} 8}=288-435^{* *} 1 / \mathrm{zn}_{\text {ttp }}$ & $\forall 4,2 \leq \mathrm{zn}_{\mathrm{tpp}} \leq 22 \mathrm{~g} \mathrm{ha}^{-1}$ & 0,337 \\
\hline
\end{tabular}

${ }^{1}$ Não houve ajuste de nenhum modelo matemático para o conteúdo de $\mathrm{S}$ nos grãos e Fe nas vagens em função do conteúdo destes nutrientes no terceiro trifólio com pecíolo, nos estádios de desenvolvimento R8 e R6, respectivamente. 
desenvolvimento R2. Restringindo-se o exemplo em questão para o fósforo, e considerando-se $3,0 \mathrm{~g} \mathrm{~kg}^{-1} \mathrm{de} \mathrm{P}$ o teor médio do nutriente na folha índice, na faixa definida como suficiente para a soja (Kurihara, 2004), tem-se que, no estádio de florescimento pleno, há um acúmulo de 1,092 $\mathrm{kg} \mathrm{ha}^{-1}$ de P neste órgão (Tabela 7). Com este valor, consegue-se estimar o máximo conteúdo de $\mathrm{P}$ no caule, nos pecíolos, nos trifólios, nas vagens e nos grãos, a partir dos modelos apresentados nas Tabelas 2 a 5, o que resul- ta em 6,$2 ; 2,3,5,7 ; 4,6$ e 24,0 $\mathrm{kg} \mathrm{ha}^{-1}$, respectivamente (Tabela 7). Em suma, seria necessário o aporte de $42,8 \mathrm{~kg}$ ha${ }^{1}$ de $\mathrm{P}$ (ou 98,0 $\mathrm{kg} \mathrm{ha}^{-1}$ de $\mathrm{P}_{2} \mathrm{O}_{5}$ ), para que a planta consiga atingir a produtividade esperada, considerando-se que os demais fatores de produção, bióticos e abióticos, não sejam limitantes. Caso a disponibilidade de P no solo não seja limitante e se deseje apenas repor a quantidade de nutriente exportado pelos grãos, então o aporte seria de 24,0 ou $54,9 \mathrm{~kg} \mathrm{ha}^{-1}$ de $\mathrm{P}$ ou $\mathrm{P}_{2} \mathrm{O}_{5}$, respectivamente. Partin-

Tabela 6. Valores médios dos coeficientes de utilização biológica (CUB) de macro e micronutrientes em parte aérea (caule, pecíolos, folhas e vagens) e grãos de soja e seus respectivos desvios padrões

\begin{tabular}{|c|c|c|c|c|c|c|}
\hline \multirow[b]{3}{*}{ Nutriente } & \multicolumn{4}{|c|}{ Parte aérea } & \multicolumn{2}{|c|}{ Grãos } \\
\hline & \multicolumn{2}{|c|}{$\ldots-\ldots R 4 \ldots$} & \multicolumn{2}{|c|}{ - - - R6 - - - } & \multicolumn{2}{|c|}{$\ldots \ldots$ R8 - . - - } \\
\hline & Média & $\begin{array}{l}\text { Desvio } \\
\text { padrão }\end{array}$ & Média & $\begin{array}{l}\text { Desvio } \\
\text { padrão }\end{array}$ & Média & $\begin{array}{l}\text { Desvio } \\
\text { padrão }\end{array}$ \\
\hline & $\ldots \ldots$ & $\ldots$ & $\ldots \ldots \mathrm{k}$ & -1 & 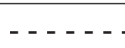 & - \\
\hline $\mathrm{N}$ & 36,9 & 3,1 & 48,9 & 6,2 & 16,3 & 0,7 \\
\hline $\mathrm{P}$ & 415,9 & 100,8 & 480,0 & 138,1 & 169,8 & 16,0 \\
\hline K & 51,9 & 10,0 & 86,6 & 17,4 & 72,7 & 18,3 \\
\hline $\mathrm{Ca}$ & 109,9 & 18,5 & 114,5 & 25,2 & 409,0 & 62,8 \\
\hline $\mathrm{Mg}$ & 269,0 & 29,8 & 303,3 & 38,4 & 372,1 & 25,5 \\
\hline S & 675,2 & 88,8 & 711,2 & 168,8 & 154,8 & 11,6 \\
\hline B & 42.676 & 5.664 & 60.358 & 15.255 & 38.735 & 4.973 \\
\hline $\mathrm{Cu}$ & 89.868 & 23.429 & 81.590 & 31.797 & 37.086 & 24.027 \\
\hline $\mathrm{Fe}$ & 8.123 & 3.504 & 11.315 & 3.651 & 12.891 & 1.326 \\
\hline Mn & 25.712 & 10.224 & 30.060 & 13.996 & 31.646 & 5.537 \\
\hline $\mathrm{Zn}$ & 42.638 & 14.372 & 56.562 & 24.756 & 15.792 & 3.577 \\
\hline
\end{tabular}

Tabela 7. Teores médios de nutrientes na folha índice (FI) na faixa definida como suficiente para a soja e quantidade acumulada de nutriente estimada para folha índice, caule, pecíolos, trifólios, vagens e grãos, para o potencial produtivo de 4 t ha-1 de grãos, por meio de modelos preditivos

\begin{tabular}{|c|c|c|c|c|c|c|c|c|}
\hline \multirow{2}{*}{$\begin{array}{l}\text { Nutri- } \\
\text { ente }\end{array}$} & \multirow{2}{*}{$\begin{array}{c}\text { Teor } \\
\text { médio }^{1}\end{array}$} & \multicolumn{7}{|c|}{ Quantidade acumulada de nutriente } \\
\hline & & $\mathrm{FI}^{2,3}$ & Caule $^{4}$ & Pecíolos $^{4}$ & Trifólios ${ }^{4}$ & Vagens $^{4}$ & Grãos ${ }^{4}$ & Total \\
\hline & $\mathrm{g} \mathrm{kg}^{-1}$ & \multicolumn{7}{|c|}{$-\ldots \mathrm{kg} \mathrm{ha}^{-1}$} \\
\hline $\mathrm{N}$ & 39,9 & 14,302 & 40,8 & 13,9 & 94,4 & 31,0 & 235,0 & 415,1 \\
\hline $\mathrm{P}$ & 3,0 & 1,092 & 6,2 & 2,3 & 5,7 & 4,6 & 24,0 & 42,8 \\
\hline $\mathrm{K}$ & 21,9 & 7,858 & 39,3 & 31,2 & 23,1 & 3,8 & 54,4 & 151,8 \\
\hline $\mathrm{Ca}$ & 10,3 & 3,687 & 12,7 & 16,7 & 24,8 & 10,0 & 9,1 & 73,3 \\
\hline $\mathrm{Mg}$ & 3,7 & 1,325 & 8,9 & 4,7 & 5,1 & 7,2 & 11,4 & 37,3 \\
\hline \multirow[t]{2}{*}{$\mathrm{S}$} & 2,6 & 0,913 & 3,3 & 0,8 & 5,0 & 2,1 & - & - \\
\hline & $\mathrm{mg} \mathrm{kg}^{-1}$ & \multicolumn{7}{|c|}{$-\mathrm{n}_{\mathrm{n}}$} \\
\hline B & 41,5 & 14,857 & 60,4 & 31,6 & 61,1 & 58,5 & 108,9 & 320,5 \\
\hline $\mathrm{Cu}$ & 8,0 & 2,864 & 40,9 & 7,3 & 10,1 & 7,7 & 121,9 & 187,9 \\
\hline $\mathrm{Fe}$ & 86,0 & 30,788 & 151,7 & 77,4 & 546,8 & - & 326,1 & - \\
\hline $\mathrm{Mn}$ & 51,0 & 18,258 & - & - & 161,8 & 44,5 & 128,6 & - \\
\hline $\mathrm{Zn}$ & 50,5 & 18,079 & 36,1 & 20,4 & 66,1 & 46,2 & 263,9 & 432,7 \\
\hline
\end{tabular}

\footnotetext{
1 Teores médios estabelecidos por Kurihara (2004); ${ }^{2}$ folha índice: terceiro trifólio com pecíolo, coletado no estádio de desenvolvimento $\mathrm{R} 2 ;^{3}$ cálculo efetuado considerando-se os teores médios estabelecidos por Kurihara (2004) e a produção estimada de $0,358 \mathrm{t}$ ha ${ }^{-1}$ de matéria seca de terceiro trifólio com pecíolo, no estádio de desenvolvimento R2, a partir da equação $\hat{\mathrm{t}}_{\mathrm{t}}=0,044 \mathrm{e}^{0,524 * * \mathrm{rg}}$, apresentada na Tabela 1 ; ${ }^{4}$ cálculo efetuado, considerando-se as quantidades acumuladas na folha índice e as equações de regressão apresentadas nas Tabelas 2 a 5 . Para os nutrientes S, Fe e Mn não foi feita estimativa de valores para todos os órgãos da planta, em decorrência da falta de ajuste de modelo de regressão.
}

Rev. Ceres, Viçosa, v. 60, n.5, p. 690-698, set/out, 2013 
do-se do mesmo princípio, estimou-se a quantidade dos demais nutrientes extraídos e exportados, para o potencial produtivo de 4,0 tha-1 (Tabela 7). Constatou-se que os macronutrientes extraídos em maior quantidade são o $\mathrm{Ne}$ o K, seguidos de Ca, P e Mg $\left(415,152,73\right.$, 43 e $37 \mathrm{~kg} \mathrm{ha}^{-1}$, respectivamente). Quanto à exportação pelos grãos, obtiveram-se as sequências $\mathrm{N}>\mathrm{K}>\mathrm{Ca}>\mathrm{P}>\mathrm{Mg}(235,54,24,11 \mathrm{e}$ $9 \mathrm{~kg} \mathrm{ha}^{-1}$, respectivamente), para os macronutrientes, e $\mathrm{Fe}>\mathrm{Zn}>\mathrm{Mn}>\mathrm{Cu}>\mathrm{B}\left(326,264,129,122\right.$ e $109 \mathrm{~g} \mathrm{ha}^{-1}$, respectivamente), para os micronutrientes.

Salienta-se, contudo, que estas estimativas de necessidade de aporte de nutrientes referem-se apenas às quantidades requeridas pelas plantas, para a reposição do nutriente extraído pela parte aérea e exportado pelos grãos. Para a definição da quantidade do nutriente, que deve ser aplicado no cultivo da soja, Santos et al. (2008) propõem que se leve em consideração a estimativa da quantidade requerida para manter a fertilidade do solo e garantir uma produtividade mínima dos cultivos subsequentes; da taxa de recuperação, pela planta, do nutriente aplicado ao solo como fertilizante, considerando-se que a planta não absorve $100 \%$ do nutriente aplicado, por causa de fatores como perdas e competição da planta com o solo; da capacidade de suprimento pelo solo, que depende da disponibilidade do nutriente, obtida a partir da análise de solo, da taxa de recuperação pelo extrator químico utilizado e do volume de solo explorado pelo sistema radicular; e da capacidade de fornecimento pelos resíduos orgânicos, a qual é dependente dos teores na matéria seca, produtividade e taxa de mineralização.

Por outro lado, considerando-se a produção de 8,259 t $\mathrm{ha}^{-1}$ de parte aérea, estimada para o estádio de desenvol- vimento R6, conforme mencionado anteriormente, a produtividade de 4,0 t ha-1 de grãos, e utilizando-se os valores médios de CUB para P (Tabela 6) de 480 e 169,8 kg grãos $\mathrm{kg}^{-1}$ de nutriente acumulado, respectivamente, temse o acúmulo de 17,2 e 23,6 kg ha-1 de P, respectivamente (Tabela 8). Ou seja, usando-se este critério de cálculo, para o potencial produtivo anteriormente mencionado, há uma extração de 40,8 $\mathrm{kg} \mathrm{ha}^{-1}$ de $\mathrm{P}$ (ou 93,4 $\mathrm{kg} \mathrm{ha}^{-1}$ de $\mathrm{P}_{2} \mathrm{O}_{5}$ ) e uma exportação pelos grãos de $23,6 \mathrm{~kg} \mathrm{ha}^{-1} \mathrm{de} \mathrm{P}$ (ou 54,0 $\mathrm{kg} \mathrm{ha}^{-1}$ de $\mathrm{P}_{2} \mathrm{O}_{5}$ ). Estes valores são muito próximos dos calculados a partir do acúmulo na folha índice (98,0 e 54,9 $\mathrm{kg} \mathrm{ha}^{-1}$ de $\mathrm{P}_{2} \mathrm{O}_{5}$, respectivamente). Para os demais nutrientes, as quantidades extraídas e exportadas (Tabela 8), foram estimadas, considerando-se o valor médio de CUB nos estádios de desenvolvimento de máximo acúmulo na parte aérea (R4, para N, K, B e Fe e R6, para Ca, Mg, S, Cu, Mn e Zn, conforme Kurihara, 2004), e a produção de 5,923 e 8,259 t ha $^{-1}$ de matéria seca de parte aérea de soja, nos estádios R4 e R6, respectivamente. Os resultados, apresentados na Tabela 8 , indicam que, à semelhança do verificado para $\mathrm{P}$, há adequada aproximação em relação aos valores calculados a partir dos modelos preditivos. Desta forma, constata-se que, apesar de os modelos de regressão apresentados nas Tabelas 2 a 5, nem sempre, apresentarem valores elevados de coeficiente de determinação, são válidos, como primeira aproximação, na consolidação do desenvolvimento de um sistema preditivo das quantidades de nutrientes necessárias à obtenção do potencial produtivo estabelecido, para a soja.

Considerando-se os valores máximos calculados a partir do acúmulo na folha índice (Tabela 7) e do valor médio de CUB (Tabela 8), pode-se definir, para um poten-

Tabela 8. Quantidade acumulada de nutriente estimada para parte aérea (caule, pecíolos, folhas e vagens) e grãos de soja, para o potencial produtivo de $4 \mathrm{t}_{\mathrm{t}} \mathrm{a}^{-1}$ de grãos, em função dos valores médios de coeficiente de utilização biológica (CUB) dos nutrientes

\begin{tabular}{lcrc}
\hline Nutriente & Parte aérea $^{1}$ & Grãos $^{2}$ & Total \\
\hline $\mathrm{N}$ & 160,5 & 245,4 & 405,9 \\
$\mathrm{P}$ & 17,2 & 23,6 & 40,8 \\
$\mathrm{~K}$ & 114,1 & 55,0 & 169,1 \\
$\mathrm{Ca}$ & 72,1 & 9,8 & 81,9 \\
$\mathrm{Mg}$ & 27,2 & 10,7 & 37,9 \\
$\mathrm{~S}$ & 11,6 & 25,8 & 37,4 \\
& $\ldots-\ldots \mathrm{kg} \mathrm{ha}^{-1}$ & $\mathrm{~g} \mathrm{ha}$ & -1 \\
$\mathrm{~B}$ & 138,8 & 103,3 & 242,1 \\
$\mathrm{Cu}$ & 101,2 & 107,9 & 209,1 \\
$\mathrm{Fe}$ & 729,2 & 310,3 & $1.039,5$ \\
$\mathrm{Mn}$ & 274,8 & 126,4 & 401,2 \\
$\mathrm{Zn}$ & 146,0 & 253,3 & 399,3 \\
\hline
\end{tabular}

${ }^{1}$ Cálculo efetuado, considerando-se o valor médio de CUB, expresso em termos de $\mathrm{kg}$ de matéria seca, produzida por kg de nutriente acumulado, nos estádios de desenvolvimento de máximo acúmulo na parte aérea (R4, para N, K, B e Fe e R6, para P, Ca, Mg, S, Cu, Mn e Zn, conforme Kurihara, 2004); também se considerou a produção de 5,923 e 8,259 t ha-1 de matéria seca de parte aérea de soja, nos estádios R4 e R6, respectivamente, estimadas a partir das equações $\quad=0,506 \mathrm{e}^{0,615^{* *} \mathrm{rg}}$ e $\hat{p}_{6}=0,717 \mathrm{e}^{0,611^{* *} \mathrm{rg}}$, respectivamente, apresentadas na Tabela 1; ${ }^{2}$ cálculo efetuado, considerando-se o valor médio de CUB e a produção de $4 \mathrm{t}$ ha ${ }^{-1}$ de grãos. 
cial produtivo de 4,0 t ha ${ }^{-1}$ de grãos, a extração de 415,169 , $82,43,38$ e $37 \mathrm{~kg} \mathrm{ha}^{-1}$ de N, K, Ca, P, Mg e S, respectivamente. Também se pode estabelecer a extração de 1.040, 433, 401, 320 e $209 \mathrm{~g} \mathrm{ha}^{-1}$ de Fe, Zn, Mn, B e Cu, respectivamente. Calcula-se, ainda, que sejam exportados pelos grãos, 245, 55, 26, 24, 11 e $10 \mathrm{~kg} \mathrm{ha}^{-1} \mathrm{de} \mathrm{N}, \mathrm{K}, \mathrm{S}, \mathrm{P}, \mathrm{Mg}$ e Ca, respectivamente, e de 326, 264, 129, 122 e $109 \mathrm{~g} \mathrm{ha}^{-1} \mathrm{de} \mathrm{Fe}$, $\mathrm{Zn}, \mathrm{Mn}, \mathrm{Cu}$ e B, respectivamente.

As informações obtidas neste trabalho complementam ou ratificam algumas premissas adotadas por Santos $e t$ al. (2008), no desenvolvimento de um sistema de recomendação de corretivos e fertilizantes para soja, junto ao Departamento de Solos da Universidade Federal de Viçosa, denominado FERTCALC ${ }^{\circledR}$ Soja.

\section{CONCLUSÕES}

A demanda nutricional pela planta de soja pode ser estimada por meio de modelos matemáticos, usando-se como base a quantidade de matéria seca produzida nos diferentes órgãos, em função do potencial produtivo almejado para a lavoura.

O produto da massa de matéria seca da folha índice (terceiro trifólio com pecíolo) com os teores de nutrientes considerados como suficientes para a cultura, resulta no conteúdo nutricional desta que, por sua vez, apresenta relação direta com o conteúdo nutricional no caule, nos pecíolos, nos trifólios, nas vagens e nos grãos.

A quantidade de nutrientes imobilizada na planta de soja também pode ser calculada a partir do quociente entre a produção de matéria seca de parte aérea e os valores de coeficiente de utilização biológica (CUB) estabelecidos.

Há adequada aproximação em relação à estimativa da quantidade de nutrientes extraídos e exportados pela soja, calculados por ambos os métodos propostos.

A partir dos dois métodos propostos para o cálculo da demanda nutricional pela planta de soja, pode-se definir, para um potencial produtivo de $4,0 \mathrm{tha}^{-1}$ de grãos, a extração de 415, 169, 82, 43, 38 e $37 \mathrm{~kg} \mathrm{ha}^{-1}$ de N, K, Ca, P, Mg e $\mathrm{S}$, respectivamente, e de 1.040, 433, 401, 320 e $209 \mathrm{~g} \mathrm{ha}^{-1} \mathrm{de}$ $\mathrm{Fe}, \mathrm{Zn}, \mathrm{Mn}, \mathrm{B}$ e Cu , respectivamente. Calcula-se, ainda, que sejam exportados pelos grãos, 245, 55, 26, 24, 11 e 10 $\mathrm{kg} \mathrm{ha}^{-1}$ de N, K, S, P, Mg e Ca, respectivamente, e de 326, $264,129,122$ e $109 \mathrm{~g} \mathrm{ha}^{-1} \mathrm{de} \mathrm{Fe}, \mathrm{Zn}, \mathrm{Mn}, \mathrm{Cu}$ e B, respectivamente.

\section{REFERÊNCIAS}

Alvarez V VH, Novais RF de, Barros NF de, Cantarutti RB \& Lopes AS (1999) Interpretação dos resultados das análises de solos. In: Ribeiro AC, Guimarães PTG \& Alvarez V VH (Eds.) Recomendações para o uso de corretivos e fertilizantes em Minas Gerais - $5^{\text {a }}$ aproximação. Viçosa, Comissão de Fertilidade do Solo do Estado de Minas Gerais. p.25-32.
Barros NF, Novais RF, Teixeira JL \& Fernandes Filho EI (1995) Nutricalc 2.0 - sistema para cálculo del balance nutricional y recomendación de fertilizantes para el cultivo de eucalipto. Bosque, 16:129-131.

Bianco S, Pitelli RA \& Carvalho LB de (2007) Estudo comparativo do acúmulo de massa seca e macronutrientes por plantas de Glycine Max (L.) Merr. e Euphorbia heterophylla L. Ensaios e Ciência, 11:61-72.

Cruz TV da, Peixoto CP, Martins MC, Brugnera A, Ledo CA da S \& Lopes PVL (2010) Acúmulo de matéria seca e área foliar de cultivares de soja em duas épocas de semeadura no Oeste da Bahia. Magistra, 22:103-111.

Fehr WR \& Caviness CE (1977) Stages of soybean development. Ames, Iowa State University. 12p.

Kurihara CH, Alvarez V VH, Neves JCL, Novais RF de \& Rodrigues DT (2003) Determinação de nutrientes em trifólios de soja por espectrometria de emissão ótica em plasma induzido. In: $29^{\circ}$ Congresso Brasileiro de Ciência do Solo, Ribeirão Preto. Anais, UNESP. CD-ROM.

Kurihara CH (2004) Demanda de nutrientes pela soja e diagnose de seu estado nutricional. Tese de Doutorado. Universidade Federal de Viçosa, Viçosa. 100p.

Lazarini E, Sá ME de \& Ferreira RC (2000) Acúmulo de matéria seca em plantas de soja durante os estádios reprodutivos e qualidade fisiológica de sementes colhidas em diferentes fases do desenvolvimento. Revista Brasileira de Sementes, 22:153-162.

Martinez HEP, Carvalho JG de \& Souza RB de (1999) Diagnose foliar. In: Ribeiro AC, Guimarães PTG \& Alvarez V VH (Eds.) Recomendações para o uso de corretivos e fertilizantes em Minas Gerais - $5^{\text {a }}$ aproximação. Viçosa, Comissão de Fertilidade do Solo do Estado de Minas Gerais. p.143-168.

Novais RF de \& Smyth TJ (1999) Fósforo em solo e planta em condições tropicais. Viçosa, Universidade Federal de Viçosa. $399 \mathrm{p}$.

Oliveira FHT de, Novais RF, Alvarez V VH \& Cantarutti RB (2005) Desenvolvimento de um sistema para recomendação de adubação para a cultura da bananeira. Revista Brasileira de Ciência do Solo, 29:131-143.

Reis Jr R dos A \& Monnerat PH (2003) DRIS norms validation for sugarcane crop. Pesquisa Agropecuária Brasileira, 38:379-385.

Santos FC, Neves JCL, Novais RF de, Alvarez V VH \& Sediyama CS (2008) Modelagem da recomendação de corretivos e fertilizantes para a cultura da soja. Revista Brasileira de Ciência do Solo, 32:1661-1674.

Serra AP, Marchetti ME, Vitorino ACT, Novelino JO \& Camacho MA (2010) Determinação de faixas normais de nutrientes no algodoeiro pelos métodos CHM, CND e DRIS. Revista Brasileira de Ciência do Solo, 34:105-113.

Silva AP da, Alvarez V VH, Souza AP de, Neves JCL, Novais RF \& Dantas JP (2009) Sistema de recomendação de fertilizantes e corretivos para a cultura do abacaxi - Fertcalc-Abacaxi. Revista Brasileira de Ciência do Solo, 33:1269-1280.

Urano EOM, Kurihara CH, Maeda S, Vitorino ACT, Gonçalves MC \& Marchetti ME (2007) Determinação de teores ótimos de nutrientes em soja pelos métodos Chance Matemática, Sistema Integrado de Diagnose e Recomendação e Diagnose da Composição Nutricional. Revista Brasileira de Ciência do Solo, 31:6372.

Rev. Ceres, Viçosa, v. 60, n.5, p. 690-698, set/out, 2013 